\title{
Palavras associadas ao câncer infantil: um levantamento exploratório
}

\section{Words associated with child cancer: an exploratory survey}

\section{Palabras asociadas al cáncer infantil: un levantamiento exploratorio}

\section{Christiane Delúsia de Oliveira Rocha*}

Universidade do Estado do Rio de Janeiro - UERJ, Rio de Janeiro, Rio de Janeiro, Brasil

\section{Lívia Gomes Viana-Meireles**}

Universidade do Estado do Rio de Janeiro - UERJ, Rio de Janeiro, Rio de Janeiro, Brasil

\section{Angela Donato Oliva***}

Universidade do Estado do Rio de Janeiro - UERJ, Rio de Janeiro, Rio de Janeiro, Brasil

\begin{abstract}
RESUMO
O diagnóstico de câncer em crianças causa um forte impacto na população, acompanhado principalmente por pensamentos negativos, apesar dos índices de cura terem aumentado nos últimos anos. Assim sendo, o presente estudo teve por objetivo analisar a percepção acerca do câncer infantil. Participaram 331 adultos, 155 com ou sem filhos e 176 pais de crianças com câncer. Para tanto, foram utilizados dois tipos de questionário: um sóciodemográfico, e outro de percepções sobre o câncer infantil. Os dados foram analisados através da estatística descritiva e uso do qui-quadrado. Os resultados indicaram que as percepções relacionadas ao câncer são predominantemente negativas. Diante disso, conclui-se que o câncer infantil está relacionado a um estigma de morte e sofrimento, o que está em contradição com os índices atuais de cura. Desse modo, o investimento no desenvolvimento de um trabalho de informação e conscientização da população pode ser importante para que o câncer seja visto de uma maneira mais realista.
\end{abstract}

Palavras-chave: câncer infantil, percepção, pais, população.

\section{ABSTRACT}

The diagnosis of cancer in children has a strong impact on the population and it is accompanied mainly by negative thoughts, although cure rates have increased in recent years. Therefore, the present study aimed to analyze the perception about childhood cancer. There were 331 adults, 155 with or without children and 176 parents of children with cancer. A sociodemographic questionnaire and a questionnaire on child cancer perceptions were used. Data were analyzed through descriptive statistics 
and chi-square use. The results indicated that perceptions related to cancer are predominantly negative. Therefore, it is concluded that childhood cancer is related to a stigma of death and suffering, which is in contradiction with current rates of cure. Thus, investing in the development of information and population awareness work may be important for cancer to be seen in a more realistic way.

Keywords: childhood cancer, perception, parents, population.

\begin{abstract}
RESUMEN
El diagnóstico del cáncer infantil causa un fuerte impacto en la población y está asociado, sobre todo, a los pensamientos negativos, a pesar del aumento de los índices de curación en los últimos años. Este estudio tuvo por objetivo analizar la percepción sobre el cáncer infantil. Participaron de la investigación 331 adultos, siendo 155 con o sin hijos y 176 padres de niños con cáncer. Fueron utilizados un cuestionario sociodemográfico y otro de percepciones sobre el cáncer infantil. Los datos fueron analizados a través de la estadística descriptiva y del uso de qui-quadrado. Los resultados indicaron que las percepciones relacionadas al cáncer son predominantemente negativas. Se concluye que el cáncer infantil está relacionado a un estigma de muerte y sufrimiento, lo que es contrario a los índices actuales de curación. Así, la inversión en el desarrollo de un trabajo de información y concienciación de la población puede ser importante para que el cáncer sea entendido de una manera más realista.
\end{abstract}

Palabras clave: el cáncer infantil, percepción, padres, población.

\title{
1 I ntrodução
}

No cenário atual, o câncer infantil é um tema que vem despertando interesse de pesquisadores de diversas áreas. Em parte, pode-se pensar que tal interesse esteja relacionado ao aumento do número de casos da doença em todo o mundo, inclusive no Brasil. Ao mesmo tempo em que a doença se expande, Couto et al. (2010) apontam que há um crescente investimento no desenvolvimento das ciências e da tecnologia e um maior acesso aos serviços de saúde. Fato que faz com que o índice de cura também tenha aumentado, podendo chegar a um patamar de $80 \%$ no Brasil (INCA, 2015). No entanto, o diagnóstico é difícil e muitas vezes desesperador já que há um forte estigma sobre a doença e este possui grande repercussão social.

Na última estimativa publicada pelo Instituto Nacional de Câncer (INCA), a previsão para o ano de 2016 no Brasil foi de aproximadamente 12.600 novos casos em crianças e adolescentes até 19 anos. As maiores ocorrências foram estimadas para as regiões Sudeste e Nordeste, com ocorrência de 6.050 e 2.750 casos respectivamente, seguidos pelas regiões sul com 1.320, Centro-Oeste com 1.270 e Norte com 1.210 casos (INCA, 2015). Já o número de óbitos no ano de 2013 chegou a cerca de 8\%, representando a segunda causa de morte entre crianças e adolescentes. É importante considerar que de acordo com o censo de 2010, 30\% da população brasileira têm até 19 anos e a patologia tem se tornado cada vez 
mais comum nessa população. Além disso, a doença possui grande repercussão social.

Mesmo com o índice de cura em torno de 80\%, a doença frequentemente remete a ideia de morte e sofrimento e é carregada de metáforas que possuem um impacto significativo na maioria das pessoas (Beltrão, Vasconcelos, Pontes, \& Albuquerque, 2007; Nehmy, Brito, Mota \& Oliveira, 2011). Isso ocorre, pois, além dos aspectos biológicos e epidemiológicos, também estão envolvidos aspectos emocionais, culturais, sociais e espirituais (Malta, Schall, \& Modena, 2009; Santos \& Gonçalves, 2008).

Além disso, a percepção sobre o câncer infantil existe independentemente do fato de que a pessoa tenha um filho com a doença, pois está ligada a forma como o sujeito aprende a responder as contingências do mundo ao seu redor, seja em aspectos diretamente relacionados à sua vivência ou aspectos sociais não relacionados diretamente à sua vida (Tourinho, Teixeira, \& Maciel, 2000). Quando é uma criança que está enferma, os valores culturais que normalmente são associados à morte possuem uma carga simbólica maior (Nehmy et al., 2011). A palavra câncer possui um estigma tão forte que o estudo de Quintana, Wottrich, Camargo e Cherer (2013) mostrou que os pais buscavam denominações mais suaves para se referir a doença.

Este estigma faz com que o recebimento do diagnóstico seja estressante e coberto de incertezas, levando os familiares à vivência de um processo doloroso (Alves, Guirardello, \& Kurashima, 2013; Beltrão, Vasconcelos, Pontes \& Albuquerque, 2007; Tarr \& Pickler, 1999). Tendo em vista o impacto causado pela doença, Popp, Conway e Pantaleao (2015) avaliaram a experiência de pais de crianças com câncer, no qual os resultados apontaram que $44 \%$ dos participantes estavam irresolutos em relação ao diagnóstico, independentemente do tempo, número de internações e idade do filho. O convívio com a doença que possui forte impacto social, e consequente preocupação acerca do futuro e medo constante da morte, determina mudanças significativas na dinâmica e nas relações familiares.

$\mathrm{O}$ processo de adoecimento e o tratamento do filho podem desencadear sofrimento psíquico (Quintana et al., 2013). A rotina da criança e das pessoas envolvidas é alterada devido a episódios de internação, terapêutica agressiva (geralmente acompanhada de efeitos colaterais desagradáveis), possível afastamento de atividades sociais e escolares, incontrolabilidade de respostas clínicas e mudanças nos cuidados parentais (Castro \& Piccinini, 2002; Kohlsdorf \& Costa Junior, 2009). Assim, o câncer representa para muitos, destruição orgânica (mutilações) e possível desestruturação do sistema familiar, uma vez que os membros passam a viver em função da criança e da patologia. Isto é acrescido a longos períodos de tratamento, frequentemente invasivos, causando limitações físicas, 
emocionais e sociais, que interferem no âmbito familiar, social e emocional não só da criança como também de seus familiares (Nascimento, Monteiro, Vinhaes, Cavalcanti, \& Ramos, 2009). Essas experiências vão contribuindo para a construção de uma memória social que pode interferir nas percepções acerca da patologia, ou seja, esta vivência repercute no meio familiar, assim como em pessoas próximas, afetando de algum modo a percepção sobre a doença.

É comum entre os pais, o desenvolvimento de transtornos como estresse, depressão, ansiedade, sintomas de estresse póstraumático, bem como mudanças nas práticas educativas, na dinâmica conjugal e na satisfação com o suporte social (Cernvall, Alaie \& Essen, 2012; Herman \& Miyazaki, 2007; Johns et al., 2009; Kazak et al., 2004; Kazak, Boeving, Alderfer, Hwang, \& Reilly, 2005). Também foram relatados pelas mães de pacientes, alterações no sono, desespero, medo da morte, preocupação e tristeza (Johns et al., 2009; Silva, Andrade, Barbosa, Hoffmann, \& Macedo, 2009). Quando as crenças são mais adaptativas em relação ao estado de saúde, as habilidades cognitivas, autonomia e satisfação com cuidados ficam relacionadas positivamente com o funcionamento psicossocial (Schwartz et al., 2012). O conhecimento dos pais sobre a doença, o tratamento e os efeitos tardios é importante para que eles se tranquilizem e motivem a criança a participar do acompanhamento de forma regular (Vetsch et al., 2015) e consigam enfrentar o tratamento de forma mais segura (Quintana et al., 2013).

Para pais e familiares, o câncer infantil é uma doença que envolve inúmeros aspectos negativos (Johns et al., 2009; Schwartz et al., 2012) e as pessoas da população em geral parecem sofrer algum tipo de impacto quando são expostas a assuntos que remetem ao tema. Mesmo as estatísticas atuais demonstrando aumento nos índices de cura e o acesso a informações sobre as doenças estarem cada vez mais disponíveis, em virtude da inexistência ou pouca eficácia do tratamento em décadas anteriores, parece ainda permanecer forte a associação de câncer (infantil) e a ideia de morte ou desfecho desagradável.

Pensando no impacto psicossocial causado pela doença e o tratamento e que esse impacto pode causar implicações no processo de enfrentamento tanto dos pais quanto do filho, Rosenberg et al. (2014) desenvolveram uma pesquisa na qual buscavam conhecer a implicação psicossocial da doença nesses pais. Os resultados mostraram mais sofrimento psíquico e busca compulsiva por bebida alcoólica $39 \%$ a mais do que os pais de crianças saudáveis. Essa preocupação também foi norteadora para uma pesquisa no I rã, no qual Nikfarid, Rassouli, Bomnijad e Alavimajd (2015) indicaram que $97,7 \%$ das mães de crianças com câncer que participaram da pesquisa, apresentavam sofrimento crônico. 
Diante disso, o presente estudo tem como objetivo analisar a percepção acerca do câncer infantil em pessoas do contexto brasileiro. As perguntas norteadoras do estudo foram: Há diferença entre as percepções no grupo da população de pais com filho com câncer e a população geral? e Há diferença nas percepções em relação ao câncer infantil dentro de cada grupo?

\section{Método}

\subsection{Participantes}

Foi realizada uma pesquisa de levantamento com adultos alocados em dois grupos: um grupo formado por 155 pessoas, com ou sem filhos (G1) e outro grupo composto por 176 pais de crianças ou adolescentes (com até 20 anos de idade) diagnosticados com câncer (G2), totalizando 331 pessoas ( $G 1+G 2$ ). Os participantes do $G 1$ tinham entre 18 e 65 anos, com média de idade de 34,9 (DP =9,8), sendo $69 \%$ do sexo feminino e $31 \%$ do sexo masculino. Destes, $92 \%$ eram provenientes das regiões Nordeste e Sudeste. A escolaridade variou entre o nível fundamental completo ( $1 \%$ ) e superior completo (46\%). Quanto ao estado civil, $46 \%$ se declararam solteiros, $45 \%$ casados e $2 \%$ viúvos. Essas pessoas foram contatadas por internet, caracterizando uma amostra de conveniência.

O G2, composto por 176 pais (mãe ou pai) de crianças ou adolescentes de até 20 anos, diagnosticados com câncer, mesmo que não estivessem em tratamento ativo. Esses pais tinham entre 18 e 67 anos e a média de idade foi de $37,3(\mathrm{DP}=9,6)$, sendo $86 \%$ do sexo feminino e $14 \%$ do sexo masculino. Nesse grupo a proporção de mulheres em relação aos homens foi maior do que no $G 1$, visto que frequentemente eram as mães que acompanhavam 0 filho ao tratamento. Os participantes eram provenientes, em $99 \%$ dos casos, das regiões Nordeste e Sudeste. A escolaridade variou entre não alfabetizados $(3 \%)$ e pós-graduados $(2 \%)$, porém a maioria possuía nível fundamental incompleto (43\%). Mais da metade da amostra se declarou casada (73\%). No que se refere a escolaridade, os grupos mostraram-se não equivalentes. Os adultos com ou sem filhos (G1) apresentaram escolaridade mais elevada (a maioria possuía nível superior ou pós-graduação). Em contrapartida, no G2, a maioria dos participantes possuía nível fundamental ou médio. A tabela 1 apresenta de forma detalhada os dados sociodemográficos dos dois grupos.

No G2 (pais de filhos com câncer), o percentual de filhos e filhas foi relativamente semelhante ( $54 \%$ de meninos e $46 \%$ de meninas). A idade dos filhos variou entre um e vinte anos, com média de 9,27 anos $(D P=5,16)$. O tipo de diagnóstico foi variado e incluiu leucemia, 
linfoma e neuroblastoma. O tempo de diagnóstico, ou seja, há quanto tempo a doença foi descoberta, variou entre um mês e quatorze anos e em $40 \%$ dos casos o diagnóstico havia sido realizado em menos de um ano.

\section{TABELA 1}

Características Sóciodemográficas dos Participantes

\begin{tabular}{|c|c|c|}
\hline Variável & Grupo 1 & Grupo 2 \\
\hline \multicolumn{3}{|l|}{ Idade (anos) } \\
\hline Menos de 20 anos & 6 & 2 \\
\hline Entre 20 e 30 anos & 31 & 23 \\
\hline Entre 31 e 40 anos & 43 & 41 \\
\hline Entre 41 e 50 anos & 10 & 25 \\
\hline Entre 51 e 60 anos & 9 & 7 \\
\hline Acima de 60 anos & 1 & 2 \\
\hline \multicolumn{3}{|l|}{ Sexo } \\
\hline Masculino & 31 & 14 \\
\hline Feminino & 69 & 86 \\
\hline \multicolumn{3}{|l|}{ Escolaridade } \\
\hline Não alfabetizado & 0 & 3 \\
\hline Fundamental incompleto & 0 & 43 \\
\hline Fundamental completo & 1 & 13 \\
\hline Médio incompleto & 2 & 16 \\
\hline Médio completo & 8 & 16 \\
\hline Técnico & 1 & 1 \\
\hline Superior incompleto & 15 & 4 \\
\hline Superior completo & 46 & 2 \\
\hline Pós-graduado & 27 & 2 \\
\hline \multicolumn{3}{|l|}{ Estado civil } \\
\hline Solteiro & 46 & 13 \\
\hline Casado & 45 & 73 \\
\hline Separado & 7 & 12 \\
\hline Viúvo & 1 & 2 \\
\hline \multicolumn{3}{|l|}{ Região em que se vive } \\
\hline Nordeste & 58 & 62 \\
\hline Sudeste & 34 & 37 \\
\hline Sul & 2 & 0 \\
\hline Norte & 3 & 1 \\
\hline Centro-oeste & 3 & 0 \\
\hline
\end{tabular}

a. Participantes Grupo $1(n=155)$ e Grupo $2(n=176)$ 


\subsection{I nstrumentos}

- Questionário Sócio demográfico: caracterizou os participantes quanto às seguintes variáveis: sexo, idade, escolaridade, estado civil e estado de origem. Os pais de crianças com câncer ainda responderam questões relativas às características sociodemográficas de seus filhos, tais como sexo, idade e tempo de diagnóstico.

- Questionário de percepções sobre o câncer infantil: teve como finalidade obter informações a respeito do que as pessoas pensam sobre o câncer infantil. Este questionário foi composto por quatro questões abertas: (1) Quando se fala em câncer, qual a primeira palavra que vem à sua cabeça? (2) Diga objetivamente, quais tipos de câncer infantil você conhece? (3) Você acha que câncer infantil tem cura? (4) Você acha que o tratamento (em crianças) é eficaz?

\subsection{Procedimento}

O estudo foi submetido e aprovado pelo Comitê de Ética em Pesquisa (CEP) da Universidade do Estado do Rio de Janeiro, sob o Parecer no 843.558. A aplicação dos instrumentos na população geral (G1) foi realizada através de uma versão online e disponibilizada em uma rede social, através de um link da plataforma de livre acesso do Google docs. Essa forma de aplicação foi escolhida para que se pudesse alcançar um número maior de participantes. Após ler as informações e os objetivos da pesquisa, as pessoas aceitavam ou não participar. As telas seguintes continham o questionário sociodemográfico e o questionário que buscava acessar as percepções que os participantes tinham sobre o câncer infantil.

Devido à dificuldade de acesso online, no grupo de pais de crianças com câncer (G2) a coleta ocorreu pessoalmente. Foi solicitado que cada responsável respondesse oralmente as perguntas para a pesquisadora. Os locais da coleta foram os serviços especializados no tratamento de câncer infantil ou de adolescente: Associação Donos do Amanhã, localizada em João Pessoa (Paraíba); Casa Durval Paiva situada em Natal (Rio Grande do Norte); Associação dos Pais e Amigos dos Leucêmicos de Alagoas (APALA), localizada em Maceió (Alagoas) e Associação Amigos da Infância com Câncer (AMICCA), Casa de Apoio à Criança com Câncer Santa Teresa (CACCST) e o Instituto Nacional do Câncer (INCA), no Rio de Janeiro.

\subsection{Preparação para análise dos dados}

O primeiro passo foi a realização de uma análise descritiva dos grupos. Em seguida, as palavras associadas ao câncer foram enviadas a três juízes para que as analisassem e classificassem em negativas, positivas e neutras (conforme ilustrado na tabela 2). A 
decisão em classificá-las em categorias, foi baseada em estudos da literatura oncológica infantil em que a doença é frequentemente associada a palavras negativas (Schwartz et al., 2012; Nehmy, Brito, Mota, \& Oliveira, 2011; Johns et al., 2009; ). Como algumas palavras podem ser vistas como positivas por alguns participantes e de forma negativa por outros, propomos uma terceira categoria, denominada "neutra". Para que uma palavra pertencesse a determinada categoria, era necessário a classificação de pelo menos dois juízes.

Entre as palavras que os juízes consideraram negativas, estão Baque, Quimioterapia e Pavor; exemplo de palavras que eles classificadas como positivas, estão Perseverança, Tratamento e Confiança. Entre as palavras consideradas neutras, temos: Careca, Normal e Desafio. O índice de concordância entre os juízes foi de 70,7\%. Como informado anteriormente, nos casos de discordância, as palavras foram alocadas nas categorias considerando a maioria das repostas iguais, ou seja, duas concordâncias.

Por se tratar de dados categóricos, após a classificação das palavras, foi utilizado o Teste Qui-quadrado para a realização de comparações entre os grupos G1 e G2 e dentro de cada grupo (quando era o caso). Como cada participante dizia uma única palavra, também foi possível utilizar o qui-quadrado em um mesmo grupo para saber se havia diferença na frequência das categorias de palavras espontaneamente faladas.

\section{Resultados e discussão}

\subsection{Análise entre os grupos}

A partir das respostas dos 331 participantes, foram contabilizadas 71 palavras diferentes, registradas as frequências com que apareciam, calculado o percentual e agrupadas nas categorias positivas, negativas e neutras de acordo com a classificação dos juízes (ver tabela 2). As palavras negativas representaram $87 \%$ das citadas pelo grupo de adultos com ou sem filhos (G1) e $79 \%$ das citadas por pais de crianças com câncer (G2). A diferença entre os dois grupos não foi estatisticamente significativa $(p>0,05)$. O percentual de palavras positivas foi de $10 \%$ para o G1 e $18 \%$ para o G2. Essa diferença também não se mostrou significativa $(p>0,05)$. Nas palavras neutras, ambos os grupos apresentaram o mesmo percentual (3\%). A análise do qui-quadrado indicou que os grupos são equivalentes em relação às crenças negativas, positivas e neutras.

Após serem computados os percentuais das palavras positivas e negativas entre os dois grupos, observou-se que no grupo de adultos com ou sem filhos (G1) as três palavras mais lembradas foram: sofrimento $(25,2 \%)$, morte $(20 \%)$ e dor $(10,3 \%)$. Para o grupo de 
pais de crianças com câncer (G2) as três palavras mais faladas foram: morte $(24 \%)$, ruim $(7,4 \%)$ e tristeza $(7 \%)$. Apesar de diferirem em duas palavras, o termo morte estava presente nos dois grupos e apresentou um percentual bastante semelhante. Essa associação entre câncer e morte vai ao encontro de outros estudos (Nehmy et al., 2011; Santos \& Gonçalves, 2008), que indicam haver uma carga emocional e simbólica maior quando a doença ocorre em crianças.

Também foi testado se a frequência das palavras comuns aos dois grupos apresentava diferença nas categorias negativas e positivas. Em relação às palavras categorizadas como negativas, as que apresentaram maior diferença entre os grupos foram: sofrimento, medo e dor. Houve diferença significativa apenas para a palavras sofrimento $\left(X^{2}=13,333 ; g l=1, p<0,001\right)$, citada por $25 \%$ do grupo de adultos com ou sem filhos (G1) e por $5 \%$ do grupo de pais de filhos com câncer (G2). Embora o estudo de Malta et al., (2009) aponte que a ideia de sofrimento causado pela doença impacta a maioria das pessoas, para os participantes do G1, a palavra câncer está significativamente mais associada ao sofrimento do que para os participantes do G2 que convivem com a doença. Uma possibilidade para entender esse resultado é o fato de que ao se deparar com a enfermidade, os pais passam a dimensionar a situação a partir de fatos reais, ao passo que aqueles que não têm essa experiência parecem apresentar medos maiores e alimentam expectativas inflacionadas de sofrimento.

Em relação às palavras positivas, as três mais citadas pelo grupo de adultos com ou sem filhos (G1) foram: luta (4\%), superação $(1,9 \%)$ e cura $(1,3 \%)$. Já no grupo de pais de crianças com câncer (G2) as três mais citadas foram: cura $(4,5 \%)$, vitória $(3,4 \%)$ e mudança $(2,5 \%)$. Apenas a crença relacionada a cura estava presente nos dois grupos e apresentou um percentual maior no G2, mas a diferença não foi significativa. Vale destacar que a ocorrência da palavra luta remete aos achados de Quintana et al., (2013) no qual as expressões bélicas são comuns no enfretamento do câncer e remetem à ideia de uma batalha a ser vencida. 
Christiane Delúsia de Oliveira Rocha, Lívia Gomes Viana-Meireles, Angela Donato Oliva

TABELA 2

Palavras associadas ao câncer infantil

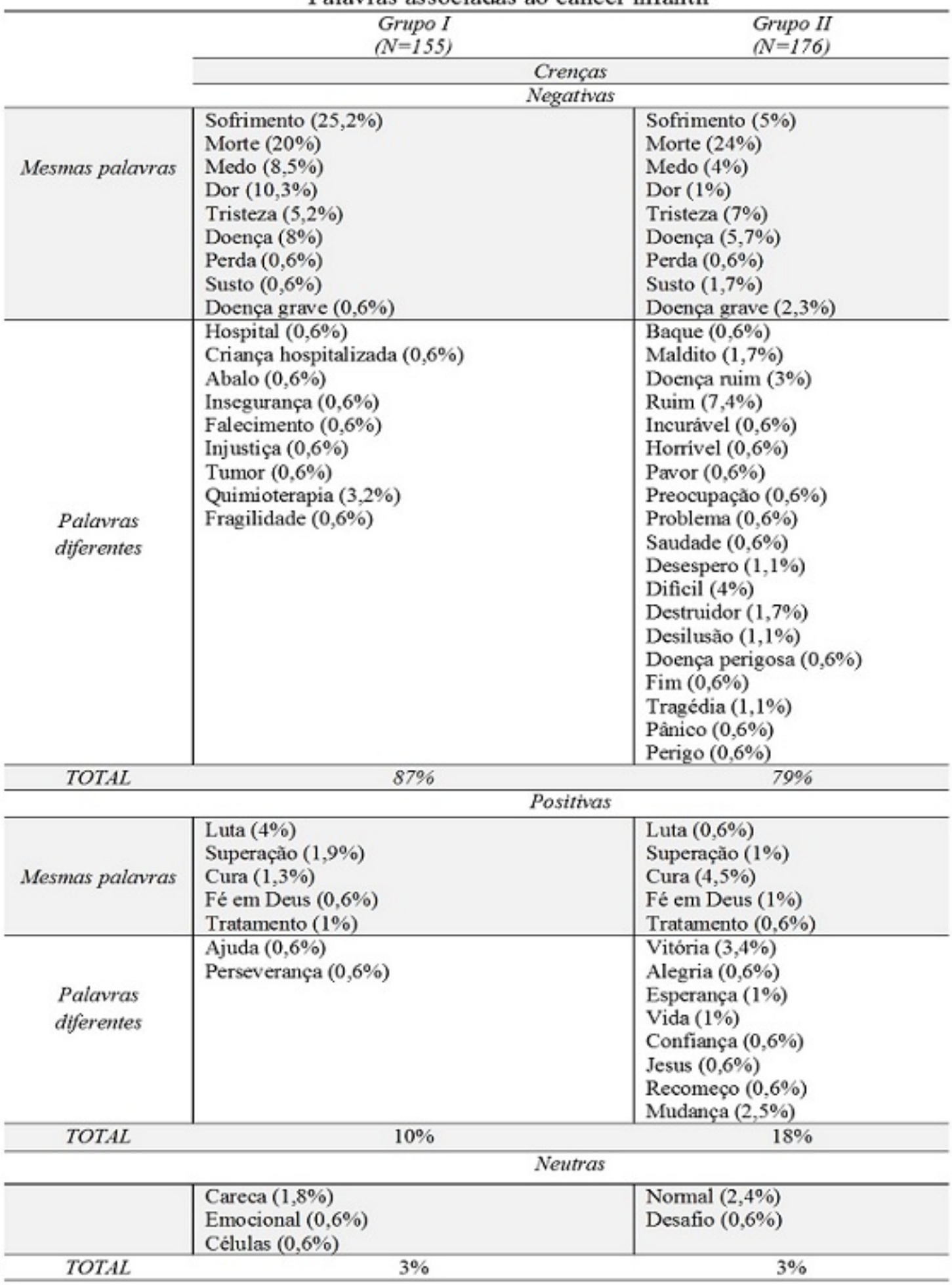

\subsection{Análise intragrupos}

Para se conhecer o perfil de cada grupo em relação às categorias de palavras citadas (negativas, positivas e neutras) foi calculado o percentual. Quando computados apenas os resultados do grupo de 
adultos com ou sem filhos (G1), observou-se que as palavras negativas somaram $87 \%$, as positivas $10 \%$ e as neutras $3 \%$. Como as palavras neutras obtiveram um percentual baixo, realizou-se 0 qui-quadrado apenas entre as categorias de palavras negativas e positivas, que equivaleram a $97 \%$ das citações. O resultado mostrou diferença significativa entre essas duas categorias $\left(X^{2}=93,781 ; \mathrm{gl}=\right.$ 1 , p $<0,001)$, ou seja, há uma predominância de palavras que denotam crenças negativas entre os adultos do G1.

No grupo de pais de crianças com câncer (G2) $79 \%$ das palavras remetiam a crenças negativas, $18 \%$ a crenças positivas e $3 \%$ a crenças neutras. Como palavras neutras também apresentaram um percentual baixo, realizou-se o qui-quadrado apenas entre as categorias negativa e positiva que somaram $97 \%$ das citações. O resultado também mostrou diferença significativa entre as duas categorias ( $\left.X^{2}=91,691 ; \mathrm{gl}=1, \mathrm{p}<0,001\right)$, com a predominância de palavras negativas.

Os resultados mostram um predomínio de palavras negativas nos dois grupos, indicando que as percepções negativas não estariam relacionadas ao fato de possuir um filho com a doença e sim ao contexto cultural. Mesmo que a maioria das pesquisas sejam realizadas com pais e familiares de pessoas com câncer é perceptível que o impacto se estende por uma parcela maior da população, ou seja, provavelmente já existiam crenças predominantes antes mesmo do diagnóstico. A associação negativa em relação à doença também foi relatada por Santos e Gonçalves (2008) indicando resultados de que as pessoas considerem o câncer como perigoso, incurável e impactante.

As análises intragrupos foram realizadas dividindo os grupos de acordo com as seguintes variáveis obtidas a partir do questionário sociodemográfico e que permitiram diferentes categorizações: sexo, estado civil, escolaridade, região e faixa etária. A escolaridade foi agrupada em três categorias: 1) pessoas não alfabetizadas e com ensino fundamental (incompleto ou completo); 2) pessoas com ensino médio (incompleto/completo) e técnico; e 3) pessoas com ensino superior (incompleto ou completo) e pós-graduação. A faixa etária também foi agrupada em três categorias: 1) entre 18 e 35 anos; 2) entre 36 e 50 anos e 3) acima de 50 anos.

\subsection{Como é o perfil daqueles que citaram as palavras classificadas como negativas}

O grupo de adultos com ou sem filhos (G1), possui o seguinte perfil: 89,4\% eram mulheres; $92,4 \%$ eram casados; $91,3 \%$ possuíam nível superior ou pós-graduação e 91,3\% tinham idade entre 18 e 35 anos. Do mesmo modo, no grupo de pais de filhos com câncer (G2): 88,6\% eram mulheres; $89,5 \%$ eram solteiros; $92,6 \%$ possuíam nível médio 
e técnico e $91,8 \%$ tinham entre 36 e 50 anos. Não foram encontradas diferenças significativas em nenhuma das categorias, contudo, dois fatos chamam atenção no G1. O primeiro é que os maiores índices de palavras negativas estão entre os participantes mais jovens, que hipoteticamente fariam parte de uma geração que não teria vivenciado diretamente épocas em que o tratamento do câncer era inexistente ou de pouca eficácia: seriam indivíduos cujo conhecimento seria de resultados promissores em relação ao câncer, com muitos tratamentos que redundam em cura. O segundo é que percentuais mais elevados de crenças negativas estão entre os participantes com alta escolaridade (graduandos e pós-graduandos), que supostamente teriam maior facilidade de acesso à informação. Esses dois aspectos de certa forma surpreenderam a expectativa que se tinha sobre a composição do perfil de pessoas dentro da categoria de palavras negativas, pois o acesso à informação sobre a alta taxa de eficácia dos tratamentos atuais não impactou na percepção da população que, supostamente, teria mais acesso a informação (jovens com alta escolaridade).

No grupo de adultos, de filhos com câncer (G2), foi feita uma análise das palavras associadas ao câncer considerando os fatores: tempo de diagnóstico, a idade do filho, e o fato de estar ou não em tratamento. Embora o tempo de diagnóstico fosse bastante variado, em $40 \%$ dos casos ele ocorreu em até um ano. Nesse grupo, foi registrado um índice de $91,2 \%$ de palavras negativas. Isso parece indicar que, apesar do fato de que o tempo de diagnóstico não apresenta diferença estatisticamente significativa, ele exerce um forte impacto na família e foi uma variável que Alves et al., (2013) associou ao desenvolvimento de estresse em cuidadores de crianças com câncer. Os autores evidenciaram que os pais que haviam recebido 0 diagnóstico há pouco tempo apresentavam maior nível de estresse e de ansiedade. Isso faz sentido com o que foi apresentado anteriormente, apontando que a experiência com a doença funciona como um aprendizado. Após o primeiro ano, há uma tendência de os pais diminuírem o grau de ansiedade e compreenderem a real evolução da doença e a eficácia do tratamento em níveis realistas, com menos medo.

Ainda no G2, a idade do filho foi previamente agrupada em três categorias: 1 ) até 6 anos; 2) entre 7 e 12 anos e 3) acima de 12 anos. A maior porcentagem de palavras negativas foi citada por pais que tinham filhos até seis anos de idade $(91,9 \%)$ e esse resultado se mostrou estatisticamente significativo $\left(X^{2}=36,938 ; \mathrm{gl}=2, \mathrm{p}<\right.$ 0,001 ) em relação às outras faixas etárias. Corroborando esse resultado, Alves et al., (2013) destacaram que crianças mais novas ainda são muito dependentes dos seus cuidadores, principalmente em relação às atividades de autocuidado, fazendo com que o impacto da doença seja maior. Quanto ao fato de que o filho ainda esteja em 
tratamento, o índice de palavras negativas foi de $66,4 \%$, porém a diferença para os que estavam apenas na fase de controle não foi significativa. Isso endossa a suposição de que a experiência ao longo do tempo favorece um conhecimento mais claro sobre o tratamento e a evolução e o controle da doença.

Quando indagados sobre seu conhecimento a respeito dos tipos de câncer, $59 \%$ dos participantes do grupo de adultos com ou sem filhos (G1) afirmaram conhecer apenas um tipo, e 8\% disseram conhecer nenhum. O tipo de câncer mais lembrado foi a leucemia (59\%), que é o mais comum entre crianças e adolescentes. No grupo de adulto que possuíam filhos com câncer (G2), 85\% dos participantes relataram conhecer apenas um tipo de câncer, ao passo que $9 \%$ disseram conhecer nenhum. Em alguns casos os pais afirmaram que só passaram a conhecer o nome de algum tipo de câncer após o diagnóstico do filho. O tipo de câncer mais lembrado no grupo G2 também foi a leucemia (40\%). Nesse grupo, chama atenção o número de pais que relatam não saber o nome de nenhum tipo de câncer, mesmo tendo um filho com a doença. Uma explicação para pode estar na menor escolaridade dos participantes deste grupo. Por outro lado, 15,3\% citaram nomenclaturas científicas mais específicas, reforçando a ideia de que a experiência funciona como aprendizagem. A crença na cura do câncer e na eficácia do tratamento, esteve presente em ambos os grupos (ver gráficos 1 e 2). Os resultados demonstraram que no grupo de adultos com ou sem filhos (G1) $93 \%$ acreditavam na cura e $97 \%$ na eficácia do tratamento. Esses altos índices também foram apresentados por pais de crianças com câncer (G2), no qual 91\% afirmaram acreditar na cura e 90\% na eficácia do tratamento. Esse resultado mostra que apesar de haver predominância de palavras negativas associadas a doença, há esperança de cura e que o tratamento oferecido possui eficácia.

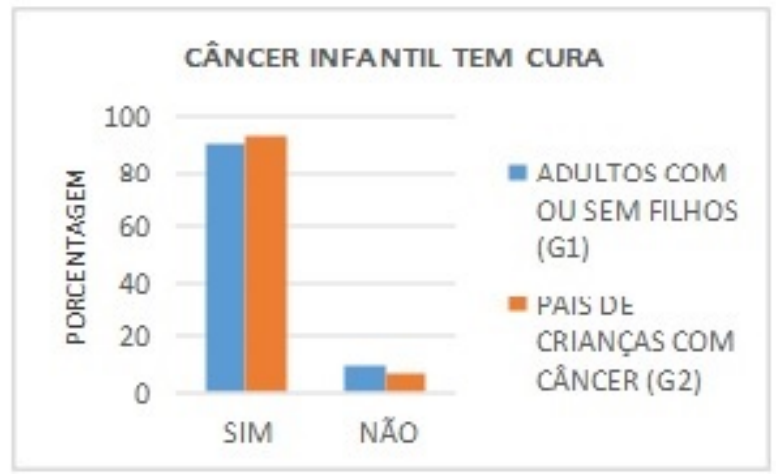

Gráfico 1: Câncer infantil tem cura 


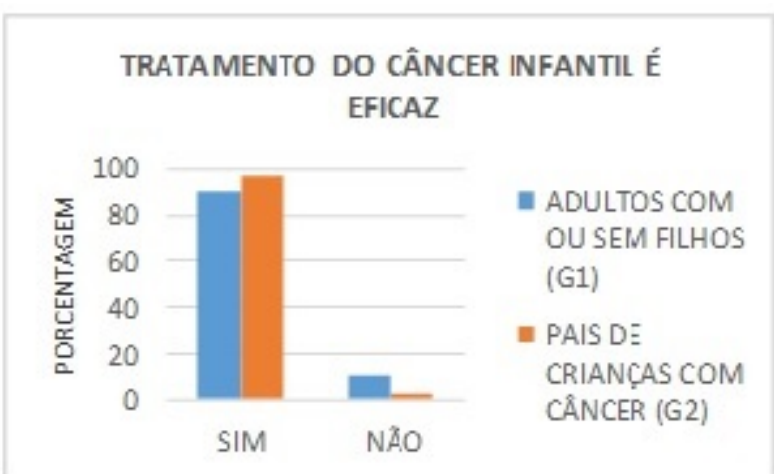

Gráfico 2: Tratamento em criança é eficaz

Percebe-se, em certo sentido, uma ambivalência nas crenças dos participantes de ambos os grupos. Por um lado, há esperança de cura, e por outro há um simbolismo significativamente negativo em relação ao câncer, no qual as crenças negativas permeiam o imaginário da maioria da população, independente de ter ou não filho com a doença. Nessa mesma perspectiva Schwartz et al., (2012) mostraram que é comum a associação de palavras negativas ao câncer, embora os avanços tecnológicos permitiram pensar em um bom prognóstico. Como a literatura evidencia, talvez as crenças negativas contribuam para o desenvolvimento de alguns transtornos psicológicos nos pais de crianças com câncer.

Após a descoberta da doença no filho, alguns pais buscam acessar informações baseadas em evidências científicas, divulgadas pelos órgãos de referência o que pode gerar uma mudança na percepção sobre a patologia. Para Beltrão et al. (2007) as mudanças de interpretação da doença podem ser reformuladas ao longo do tempo de acordo com o processo interativo. Ou seja, quanto mais a pessoa se informa, maior a sensação de controle da situação e, consequentemente, maior a força para enfrentá-la. Os resultados obtidos pelo presente estudo vão na mesma direção e sugerem que a mudança no modo como a doença é vista, pode estar mais associada ao fato de haver um contato direto com ela do que a fatores como escolaridade ou idade. Como mostra a tabela 2, há uma associação maior do câncer com palavras negativas por parte de pessoas que não tem filhos com câncer. Nesse sentido, percebe-se que no meio em que esses pais estão inseridos, há uma ideia sobre o câncer infantil e que esta pode ser mudada após o contato com a doença e busca da informação. Até que isto ocorra, os pais já estão consumidos pelo sofrimento e dor causados pelo modo como a doença é vista socialmente, o que dificulta o processo e enfrentamento. Pois, ao que parece, a experiência pode gerar um melhor conhecimento e consequentemente contribuir para a mudança nas crenças e enfrentamento. 
De um modo geral, muito embora a literatura científica informe que os índices de cura são cada vez maiores, as crenças negativas ainda são predominantes para a amostra investigada, independentemente da idade, grau de escolaridade, sexo, estado civil e região em que vive. Mesmo com todo acesso aos meios de informação, as ideias sobre a doença parecem que só mudam quando se passa pela experiência. Diante da necessidade, ocorre a busca por informação baseada em evidências quanto ao prognóstico e tratamento, e com isso as-percepções podem ser mais condizentes com a realidade e menos baseadas em suposições sobre a doença.

\section{Considerações finais}

Este estudo buscou, de uma forma inédita, saber as percepções sobre o câncer infantil, tanto de pais cujo os filhos foram diagnosticados de câncer, como da população em geral, já que as pesquisas normalmente buscam apenas o que os pais falam sobre a doença. $O$ resultado corroborou com estudos anteriores que apontam associações negativas ao câncer. No entanto, percebeu-se o percentual maior de palavras negativas associadas ao câncer, principalmente da palavra morte, na população em geral (G1). Isso pode demonstrar que havendo uma percepção prévia sobre a doença, os pais, no momento do diagnóstico podem ser prender mais ao que é socialmente compartilhado do que ao quadro real da criança.

Nesse sentido, os resultados são relevantes ao apontar para a necessidade do desenvolvimento de campanhas para a identificação precoce do câncer infantil, para que a doença seja encarada de maneira realista, de acordo com o prognóstico da criança. Há de se destacar que foi encontrada uma ambivalência quanto a percepção da doença e boas expectativas de cura e de um bom prognóstico, fato que ainda precisa ser mais bem explorado em pesquisas posteriores. Ideias equivocadas parecem contribuir no surgimento de transtornos psíquicos, o que pode dificultar o enfrentamento da doença.

Finalmente, os resultados sugerem que investir no desenvolvimento de um trabalho de informação e conscientização da população pode ser importante para que o câncer seja desmistificado e a doença passe a ser encarada de uma maneira mais realista. Ou ainda, que sejam desenvolvidos instrumentos que auxiliem na identificação dessas crenças para que elas possam ser trabalhadas de maneira realista.

Esta pesquisa propiciou uma reflexão importante acerca de como o câncer infantil é visto por pais e adultos em geral, destacando que na literatura são escassos os estudos que se propõem a identificá-las. Este conhecimento é um passo importante para auxiliar os profissionais que lidam diretamente com a família e o paciente no 
sentido de elaborar estratégias que visem auxiliar ambos nesse processo de enfrentamento. Além disso, a qualidade da relação entre o cuidador e o filho é essencial no processo de enfrentamento tanto da doença quanto do tratamento, e se o estado psicológico e qualidade de vida do cuidador estão comprometidos, podem afetar de alguma forma tanto a relação com a criança ou adolescente quanto o processo de enfrentamento do filho em relação à doença.

Este estudo possui algumas limitações, entre elas, a de que os grupos não foram totalmente equivalentes. O grupo de população geral não foi apenas de pais, o que pode ter reforçado a frequência de palavras negativas ao câncer. Não foi controlado o tempo de diagnóstico da criança nem o tipo de tratamento. Nesse sentido faz-se necessário que estudo futuros possam utilizar controlar essas variáveis.

\section{Referências}

Alves, D. F. S., Guirardello, E. B., \& Kurashima, A. Y. (2013). Estresse relacionado ao cuidado: o impacto do câncer infantil na vida dos pais. Revista Latino-Americana de Enfermagem. 21(1), 356-362. doi: http://dx.doi.org/10.1590/S010411692013000100010

Beltrão, M. R. L. R., Vasconcelos, M. G. L., Pontes, C. M., Albuquerque, M, C. (2007). Câncer infantil: percepções maternas e estratégias de enfrentamento frente ao diagnóstico. Jornal de Pediatria, 3(6), 562-566. doi: http://dx.doi.org/10.1590/S0021-75572007000800014

Castro, E. K., \& Piccinini, C. A. (2002). Implicações da doença crônica na infância para as relações familiares: algumas questões teóricas. Psicologia: Reflexão e Crítica, 15(3), 625-635. doi: http://dx.doi.org/10.1590/S0102-79722002000300016

Cernvall, M., Alaie, I., \& Essen, L. (2012). The factor structures traumatic stress in parents of children with cancer: a longitudinal analysis. Journal of Pediatric Psychology, 37(4), 448-457. doi: 10.1093/jpepsy/jsr105. Epub 2011 Dec 13.

Couto, A. C., Ferreira, J. D., Koifman, R. J., Monteiro, G. T., Pombode-Oliveira, M. S., \& Koifman, S. (2010). Trends in childhood leukemia mortality over a 25-years period. Journal of Pediatric, 86(5), 405-410. doi: 10.2223/J PED.2027.

Herman, A. R. S., \& Miyazaki, M. C. O. S. (2007). Intervenção psicoeducacional em cuidador de criança com câncer: relato de caso. Arquivos de Ciência da Saúde, 14(4), 238-244.

Instituto Nacional de Câncer [INCA] (2015). Estimativa 2016: incidência de câncer no Brasil. Rio de Janeiro: INCA.

Johns, A. L., Oland, A. A., Katz, E. R., Sahler, O. J., Askins, M. A., Butler, R. W., \& Dolgin, M. J. (2009). Qualitative analysis of the 
Role of culture in coping themes of Latina and European American Mothers of Children with Cancer. Journal of Pediatric Oncology Nursing, 26(3), 167-175. doi: 10.1177/1043454209334416. Epub 2009 Apr 27.

Kazak, A. E., Alderfer, M., Rourke, M, T., Simms, S., Streisand, R., \& Grossman, J. R. (2004). Posttraumatic Stress Disorder (PTSD) and Posttraumatic Stress Symptoms (PTSS) in Families of Adolescent Childhood Cancer Survivors. Journal of Pediatric Psychology, 29(3), 211-219. doi: 10.1093/jpepsy/jsh022

Kazak, A. E., Boeving, C. A., Alderfer, M. A., Hwang, W T., \& Reilly, A. (2005). Posttraumatic stress symptoms during treatment in parents of child with cancer. Journal of Clinical Oncology, 23(30), 7406-7410. doi: 10.1200/JCO.2005.09.110

Kohlsdorf, M., \& Costa Junior, A. L. (2009). Enfrentamento entre Cuidadores de Pacientes Pediátricos em Tratamento de Leucemia. Interação em Psicologia, 13(2), 263-274.

Malta, J. D. S., Schall. V. T., \& Modena, C. M. (2009). O momento do diagnóstico e as dificuldades encontradas pelos oncologistas pediátricos no tratamento do câncer em Belo Horizonte. Revista Brasileira de Cancerologia, 55(1), 33-39.

Nascimento, C. A. D., Monteiro, E. M. L. M., Vinhaes, A. B., Cavalcanti, L. L., \& Ramos, M. B. (2009). O câncer infantil (leucemia): significações de algumas vivências maternas. Revista da Rede de Enfermagem do Nordeste - Rev Rene, 10(2), 149-157.

Nehmy, R. M. Q., Brito, A. C. B., Mota, J. A. C., \& Oliveira, B. M. (2011). A perspectiva dos pais sobre a obtenção do diagnóstico de leucemia linfóide aguda em crianças e adolescentes: uma experiência no Brasil. Revista Brasileira de Saúde Materno Infantil, 11(3), 293-299. doi: http://dx.doi.org/10.1590/S151938292011000300010

Nikfarid, L., Rassouli, M., Borimnejad, L., \& Alavimajd, H. (2015). Chronic Sorrow in Mothers of Children With Cancer. J ournal of Pediatric Oncology Nursing, 32(5), 314-319. doi: 10.1177/1043454214563407

Popp, J. M., Conway, M., \& Pantaleao, A. (2015). Parents' Experience With Their Child's. Cancer Diagnosis: Do Hopefulness, Family Functioning, and Perceptions of Care Matter? Journal of Pediatric Oncology Nursing, 32(4), 253-260. doi: 10.1177/1043454214563404

Quintana, A. M., Wottrich, S. H., Camargo, V. P., \& Cherer, E. Q. (2013). Childhood Cancer: Meanings Attributed to the Disease by Parent Caregivers. Paidéia, 23(55), 253-261. doi: 10.1590/1982-43272355201313

Rosenberg, A. R., Wolfe, J., Bradford, M. C., Shaffer, M. L., Yi-Frazier, J. P., Curtis, R., \& Bakerm, K. S. (2014). Resilience and 
psychological outcomes in parentes of children with cancer. Pediatric Blood \& Cancer, 61(3), 552-557.

Santos, L. M. P., \& Gonçalves, L. L. C. (2008). Crianças com câncer: desvelando o significado do adoecimento atribuído por suas mães. Revista de Enfermagem UERJ, 16(2), 224-229.

Schwartz, L. A., Kazak, A. E., De Rosa, B. W., Hocking, M. C., Hobbie, L. W., \& Ginsberg, J. P. (2012). The role of beliefs in the relationship between health problems and posttraumatic stress in adolescent and young adult cancer. Journal of Clinical Psychology in Medical Settings, 19(2), 138-146. doi: 10.1007/s10880-011-9264-1.

Silva, F. A. C., Andrade, P. R., Barbosa, T. R., Hoffmann, M. V., \& Macedo, C. R. (2009). Representação do processo de adoecimento de Crianças e adolescentes oncológicos junto aos Familiares. Escola Anna Nery Revista de Enfermagem, 13(2), 334-341. doi: http://dx.doi.org/10.1590/S141481452009000200014

Tarr, J., \& Pickler, R. H. (1999). Becoming a cancer patient: a study of families of children with acute lymphocytic leukemia. Journal of Pediatric Oncology Nursing, 16(1), 44-50. doi: 10.1177/104345429901600106

Tourinho, E. Z., Teixeira, E. R. \& Maciel, J. M. (2000). Fronteiras entre análise do comportamento e fisiologia: Skinner e a temática de eventos privados. Psicologia: Reflexão e Crítica, 13(3), 425-434.

Vetsch, J., Rueegg, C. S., Gianinazzi, M. E., Bergstrãsser, E., Von der Weid, N. X., \& Michel, G. (2015). Information Needs in Parents of Long-Term Childhood Cancer Survivors. Pediatric Blood Cancer, 62(5), 859-66. doi: 10.1002/pbc.25418

\footnotetext{
Endereço para correspondência Christiane Delúsia de Oliveira Rocha

Universidade do Estado do Rio de Janeiro

Rua São Francisco Xavier, 524, 10ㅇan andar, Maracanã, CEP 20.550-900, Rio de Janeiro - RJ, Brasil

Endereço eletrônico: christiane.delusia@gmail.com

Lívia Gomes Viana-Meireles

Universidade do Estado do Rio de Janeiro

Rua São Francisco Xavier, 524, 10ㅇan andar, Maracanã, CEP 20.550-900, Rio de Janeiro - RJ, Brasil

Endereço eletrônico: liviagviana@gmail.com

Angela Donato Oliva

Universidade do Estado do Rio de Janeiro

Rua São Francisco Xavier, 524, 10ㅇ andar, Maracanã, CEP 20.550-900, Rio de Janeiro - RJ, Brasil

Endereço eletrônico: angeladonatoliva@gmail.com
} 
Recebido em: 28/01/2016

Reformulado em: 14/02/2018

Aceito em: $16 / 08 / 2018$

\section{Notas}

* Doutora em Psicologia Social pela Universidade do Estado do Rio de Janeiro.

** Doutora em Psicologia Social pela Universidade do Estado do Rio de J aneiro.

*** Professora do Departamento de Psicologia e do Programa de Pós-graduação em Psicologia Social da Universidade do Estado do Rio de Janeiro - UERJ; Doutorado em Psicologia Escolar e do Desenvolvimento Humano.

Este artigo de revista Estudos e Pesquisas em Psicologia é licenciado sob uma Licença Creative Commons Atribuição-Não Comercial 3.0 Não Adaptada. 